\title{
Improving IPv6 Wireless Ad Hoc Networks QoS via Enhanced Flow Label with Stability Based Dynamic Source Routing Scheme
}

\author{
${ }^{1}$ Wai Yee Tai, ${ }^{2}$ Chong Eng Tan, ${ }^{3}$ Sei Ping Lau \\ Department of Computer Systems and Communication Technologies, \\ Faculty of Computer Science and Information Technology , Universiti Malaysia Sarawak, \\ Malaysia \\ e-mail: ${ }^{1}$ whyee86@gmail.com, $\left\{{ }^{2}\right.$ cetan, ${ }^{3}$ splau $\} @$ fit.unimas.my
}

\begin{abstract}
This paper will present an enhanced Dynamic Source Routing (DSR) routing protocol where it utilizes the IPv6 Flow Label to provide Quality-of-Service $(\mathrm{QoS})$ to the real time applications in IPv6 wireless ad hoc networks. Conventional DSR routing protocol is enhanced to induce better QoS in dynamic nature ad hoc networks. Internet Engineering Task Force (IETF) presented Flow Label with 20 bits in the IP packet header to provide special handlings to real time transmissions where they require high QoS services in the network. Multimedia applications required low delay, low packet drop and efficient delivery. Preliminary results of the proposed scheme will be discussed in this paper where the scheme utilized the Flow Label in IPv6 packet header to reserve the resources in order to provide a better performance in the dynamic nature ad hoc network.
\end{abstract}

Keywords-component; Wireless ad hoc networks, Quality-ofService (QoS), Dynamic Source Routing, IPv6

\section{INTRODUCTION}

Wireless ad hoc network comprises multiple of nodes connected among each other wirelessly without any management on any transmissions. It is typically deployed at the site of catastrophe, during a military manoeuvre or being used for various purposes that require instant connections whereby fixed infrastructures are unavailable. Nodes in ad hoc network are self-organized freely where they create arbitrary connections dynamically among themselves as the intervening nodes in the ad hoc network mutually agreed to forward the data from the source to their respective destination.

However, disruptions in terms of jitters, delays and so much more are often present in wireless transmissions. These will degrade the whole network performance and reduced the QoS especially in transmissions that involve real time applications such as Video-on-Demand (VOD), Music-On-Demand (MOV) and so forth. Real time transmissions are required to have acceptable performance such as low delay in order to guarantee a good QoS for the end users. Regrettably, the dynamic nature of the ad hoc network causes the transmission links to break at unpredictable time hence inducing poor QoS in the transmissions. Thus, a perfect QoS for this dynamic ad hoc network is doubtful.

Conventional Internet Protocol version 4 (IPv4) provides "best-of-efforts" delivery where it does not guarantee the data packet delivery. Therefore, IPv4 is not sufficient in rendering a good QoS for a highly dynamic ad hoc network where nodes are not statically located. Internet Protocol version 6 (IPv6) is then introduced with a 20 bits field named as Flow Label that will provide special handlings to respective flows in the network. Proposed by the Internet Engineering Task Force (IETF) to alleviate the depletion of IP address in year of 1998, the Flow label is said to induce an efficient way for state lookup on flows, packet labelling and security, and with authentication capabilities that will benefit the routing process in the ad hoc network [1].

This paper presents the proposed algorithm where Dynamic Source Routing (DSR) is enhanced by introducing stability and enhanced Flow Label to provide better QoS in transmitting data packets in dynamic IPv6 ad hoc network. Section II introduces the existing ad hoc routing protocols and some related work while Section III discusses reactive routing protocol DSR overview. Section IV introduces the Flow Label in IPv6 together with the proposed algorithm, simulation results and preliminary evaluation. Section V concludes the paper.

\section{Existing Ad Hoc Routing Protocols}

Routing protocol is a protocol that stated the way of communication among routers [2] hence, ad hoc routing protocols are the protocol that specifies how the nodes in the ad hoc network should communicate among themselves. There are numerous routing protocols proposed over the years to the ad hoc networks and they are generally categorised in two which are the Table-driven (Proactive) routing protocol and the On-Demand (Active) routing protocol. 